\title{
PREFACE
}

We cannot solve our problems with the same level of thinking that created them.

- Albert Einstein

Before tweets and texts and clouds, even before "friend" was a verb, baby boomers (those born between 1946 and 1964) outnumbered everyone else in the workplace - and they were the drivers of commerce. They were the creators, the thinkers, the fixers, and the doers. They were the entrepreneurs, the CEOs, and the presidents. But what used to be isn't any more. Today, this same group of onceenergetic capitalists is often portrayed as showing up at work and coasting. What's more, many managers seem to believe it's a kindness to let them do so. Some companies ignore boomers as a rich talent resource and count on the millennials as the only people technically savvy enough to navigate today's technologically confounding workplace. But on close scrutiny, we identify boomers as revolutionaries - a population of disruptors that is altering career patterns, creating new expectations, and demanding inclusion. Indeed, this demographic is a revolutionary force - one of the five key drivers shaping the future of work. And if, as a CEO, manager, or HR professional, you want to improve productivity, fill the talent pipeline, improve intergenerational effectiveness, and maximize your competitive advantage, boomers are not to be overlooked.

The future of work is an important discussion as we come within striking distance of 2020. Some look forward with anticipation to 
the countless ways in which work will change over the next decades. Others fear a host of challenges triggered by an aging workforce, intergenerational dynamics, new working models, the need for a living wage, and, of course, artificial intelligence and automation. This book focuses primarily on the role that workplace demographics - radically altered by longevity - will play in the future of work discussion. It explains how longevity is the catalyst for the talent revolution, and how your organization can capitalize on the opportunity before it.

Every revolution has its own unique set of revolutionaries, trailblazers, casualties, circumstances, influences, and issues, and the talent revolution is no different. It is the logical consequence of a confluence of human realities and innovation that is transforming the world of work. It is nothing less than a major workplace upheaval in need of insightful leadership. The Talent Revolution illuminates what is happening in your workplace today and what is likely to occur as we look toward 2030 and the future of work.

One may well ask: What on earth is going on? Company loyalty has evaporated and the era of staying in the same job for twenty years has long passed. Indeed, it appears that workplace demographics have changed so dramatically that managing the multigenerational workforce is a nightmare. Older workers seem to clog the system and younger workers often quit soon after signing on. Companies are doing their best to urge out boomers while ingratiating themselves to millennials with games rooms, workout spaces, candy carts, and massages. The workplace is in flux and leaders are often at a loss.

What strategies must CEOs undertake to unlock the intergenerational potential within their workforces? What can managers do to supercharge the employees on the payroll? What can HR do to ensure an inclusive culture that motivates excellence at every stage and every age? How can the organization do what it does better, faster, cheaper, and more effectively than the competition? These are a few of the many questions we have been asking in our work with 
organizations big and small across North America. The book you are reading flows from what we have learned and the answers we have found. While not all our suggestions are universally applicable, the solutions we offer, the models we present, and the strategies we suggest have been battle-tested in the field, and they may provide the answers you seek. That's why we've written The Talent Revolution - to provide new insights and strategies for turning an aging workforce into a competitive advantage.

The topics of workplace change, shifting employment models, and labor strategy are hot, and they will undoubtedly trigger countless studies by a range of academics and corporate analysts, providing more than enough material to fill volumes for years to come. Our concern is more practical and immediate. As business practitioners focused on supercharging workforces and maximizing talent equity, we dig down and look more closely at the human element - now. Call us unorthodox, but we see demographics as the single greatest competitive opportunity on which smart organizations must capitalize. Furthermore, we suggest it demands urgent attention.

The workplace is a different arena than it was even a decade ago. Complicated times, complex technology, and thorny human issues have coalesced in recent years to create what often feels like a chaotic state of affairs, one that is as difficult to plan for as it is to characterize. But we see patterns in the chaos - patterns we have organized into a practical model so leaders can make sense of the dynamics now in play and effectively modify workforce strategies to capitalize on the changes they are experiencing.

We contend that the talent revolution is underway. Our work, our reading of the literature, and our own proprietary research and analysis leads us to this conclusion: the future of work is being shaped by technological and talent-driven innovation, and it can best be understood by examining five significant drivers - drivers that individually and collectively are transforming the way work gets done. We believe that the structures and cultural attitudes at play in most organizations 
are outdated and counterproductive, that they fail to take advantage of the intellectual capital currently within their ranks, that organizations can thrive with new thinking, innovative strategies, and new models, and that an attitudinal and structural reset will propel early adopters of the new models ahead of their competition. We believe this because it is already happening with our clients.

This book spotlights the revolutionary impact the changing workforce is having on today's corporations. We apply our research, findings, and recommendations to target those who must work together to navigate the shifting world of work in the context of business survival, growth, and future relevance: the CEO, concerned with new labor structures, markets, and conditions, and how these factors will accelerate or impede business strategy; HR leaders, who find themselves on the cutting edge of massive change that falls squarely within their area of responsibility; and frontline managers, who participate in career-focused, talent-related conversations with staff, often without a broader understanding of how the world of work is shifting.

We have divided the book into three parts, and we begin by making the case for joining the revolution. In part 1, we "zoom out" and offer a new model categorizing and clarifying today's workplace issues so that all three groups of leaders can take control of the revolutionary forces with which they must contend. We demonstrate that organizations have what we call a "Broken Talent Escalator ${ }^{\circledR}$ " that is, career-path structures that ensure the boomer population either exits the workforce completely or stalls on the top step during their final decades of work. We assert that there is a generally unexplored stage of work after retirement age we call the "Legacy Career ${ }^{\circledR \prime *}$ phase. And we describe the problems with the status quo and illustrate how employers and employees are missing out on a

\footnotetext{
* The terms "Broken Talent Escalator" and "Legacy Career" are registered trademarks belonging to Challenge Factory Inc. All Rights Reserved.
} 
significant opportunity to benefit from a currently undervalued work-life phase.

Part 2 addresses standard metrics and debunks the top five myths keeping companies mired in obsolete thinking, thereby hampering the development of a robust talent resource within their ranks:

- the myth of excessive salaries

- the myth of the best before date

- the myth of squandered budgets

- the myth of diminished productivity

- the myth of generational performance characteristics

While exposing the truth, we surface the underlying organizational needs that remain unmet and unacknowledged, the needs at the root of today's workforce dysfunction.

Part 3, the final section, provides actionable steps divided into segments, each applicable to a specific leadership role. It offers areas of focus and action for CEOs, HR leaders, and frontline managers, each of whom find themselves responding to different signs and symptoms of the talent revolution.

Throughout, we take an approach that advocates a deeper understanding and application of career development as a critical discipline that helps leaders tackle the challenges posed by talent management and career models. We provide stories, methods, and tools to improve performance, engagement, and intergenerational effectiveness, and we offer new plans for transforming traditional "retirement years" into a productive and meaningful work-life segment - a period during which the mature population within an organization achieves personal, organizational, and societal gains. And as we show, organizations will benefit from this transformation.

As you read, you may encounter issues of pressing concern, issues you wish to tackle immediately. This graphic directs you to applicable solutions in part 3. 
It is said that some people change their mind when they see the light, while others wait until they feel the heat. Our intention is to shine a burning light on the information you need to adjust your point of view and alter your expectations, thereby energizing your entire workforce and profiting from it. To that end, we present a new approach to understanding and managing the forces - the five drivers - at play in today's workplace.

A manufacturing company we have been working with found itself in a difficult position. With 55 percent of its employee base (and 67 percent of its leadership team) currently eligible for retirement, there was a growing realization that recent concentration on newgraduate recruitment would not address the significant knowledge and leadership gap this company would face. The succession crunch was not hard to predict. Based on the average age employees at this particular firm typically retire, as well as an understanding of the incentives built into the pension program, our analysis determined that without a significant rethinking of the employee lifecycle, and lacking new ways to capture, retain, and translate knowledge, this company would hit a wall in 2022. The uncertain timing and impact of succession is expensive and damaging to workplace culture. Helping this company understand the Broken Talent Escalator and how alumni could save their organization time and money - two solutions identified in this book - enabled the company to avoid the costly and dangerous approach of "waiting out the clock."

In another sector, a financial services firm with more than 120 years of history and culture recognized that the future of their business required a complete rethinking of core relationships. Instead of being the provider of traditional financial products and services to a relatively stable customer base, as they had been throughout their history, this firm wanted to transform itself into a flexible provider of technology platforms and services that react to new market and technology conditions. Its goal was to become a technology firm focused on the financial services market. A major transformation of 
this kind is enormously challenging, and executives wondered if their older workers were up for the task ahead. It was immediately apparent to us that engagement among senior leaders of the organization was on the decline at precisely the time the company needed strong cultural leadership from the top. Moreover, myths and stereotypes about different generations abounded. It was therefore critical that in transforming their culture, leaders acknowledged boomers' lifelong role as revolutionaries. Furthermore, leaders needed a deeper understanding of what employees in their fifties, sixties, seventies, and beyond require in order to perform maximally and continue to be engaged. The situation required a shift in core relationships, an essential part of ensuring that the old guard, the boomers, understood the new goals and were enthusiastic about helping the brand - a brand to which they had committed decades of service - thrive in this new economy.

The dialogue with senior leaders continues to evolve, moving from curiosity about workforce data to testing new ideas for workforce engagement and integrating our solutions for improved intergenerational workforce effectiveness.

Until now, there has been no manual, no useful model, and no clear pathway forward. This changes everything. We call for champions of the talent revolution. Prepare to lead! 
This page intentionally left blank 


\section{THE TALENT REVOLUTION}

LONGEVITY AND THE FUTURE OF WORK 
This page intentionally left blank 